\title{
3D Face Recognition Analysis Using Random Forest
}

\author{
NURUL ATIFAH ROSLAN, HAMIMAH UJIR* \& IRWANDI HIPNI MOHAMAD HIPINY
}

\author{
Faculty of Computer Science and Information Technology, Universiti Malaysia Sarawak, 94300 Kota Samarahan, \\ Sarawak, Malaysia \\ *Corresponding author: uhamimah@unimas.my
}

\begin{abstract}
Face recognition is an emerging field due to the technological advances in camera hardware and for its application in various fields such as the commercial and security sector. Although the existing works in 3D face recognition perform well, a similar experiment setting across classifiers is hard to find, which includes the Random Forest classifier. The aggregations of the classification from each decision tree are the outcome of Random Forest. This paper presents 3D facial recognition using the Random Forest method using the BU-3DFE database, which consists of basic facial expressions. The work using other classifiers such as Neural Network (NN) and Support Vector Machine (SVM) using a similar experiment setting also presented. As for the results, the Random Forest approach has yield 94.71\% of recognition rate, which is an encouraging result compared to NN and SVM. In addition, the experiment also yields that fear expression is unique to each human due to a high confidence rate (82\%) of subjects with fear expression. Therefore, a lower chance to be mistakenly recognized someone with a fear expression.
\end{abstract}

Keywords: 3D face recognition, Neural Network, Random Forest, Support Vector Machine

Copyright: This is an open access article distributed under the terms of the CC-BY-NC-SA (Creative Commons Attribution-Non-Commercial-Share Alike 4.0 International License) which permits unrestricted use, distribution, and reproduction in any medium, for non-commercial purposes, provided the original work of the author(s) is properly cited.

\section{INTRODUCTION}

3D face recognition is the enhancement of face recognition technology in a few aspects. It has the possibility to overcome feature localization, pose and illumination problems. 3D face recognition is known for its easy and nonintrusive acquisition of face information compared to the other biometrics. Moreover, 3D face recognition has the low privacy of information compared to signatures and fingerprints (Gökberk et al., 2008). In recent years, 3D face recognition system has become a popular biometric system due to its higher capability and accuracy, and for its application in various fields such as in commercial, security, health and banking sector.

Existing works in 3D face recognition have been using various kinds of classifiers, for example, Neural Networks (NN) (Hu et al., 2013; Kim et al., 2017), Support Vector Machine (SVM) (Mousavi et al., 2008), Principal Component Analysis (PCA) (Lee \& Han, 2006) and Linear Discriminate Analysis (Drira et al., 2012; Hiremath, 2013). These classifiers have a few issues when dealing with high dimensional data and facing an overfitting problem. Works using Random Forest also have been discussed in Salhi et al. (2012) and, Kremic and Subasi, (2016). However, the comparison with other classifiers using a similar experimental setting has not been presented in any work.

Our objectives are to develop 3D facial recognition using Random Forest and to compare Random Forest with two existing methods, which are Probabilistic Neural Network (PNN) and SVM using a similar experiment setting. In this paper, the analyses of the 3D face recognition experiments conducted are presented. Section 2 describes the existingrelated Random Forest works in this study and followed by an explanation of our work in section 3. Section 4 discusses the results and analysis of the experiments. Finally, the conclusions and future works are drawn. 